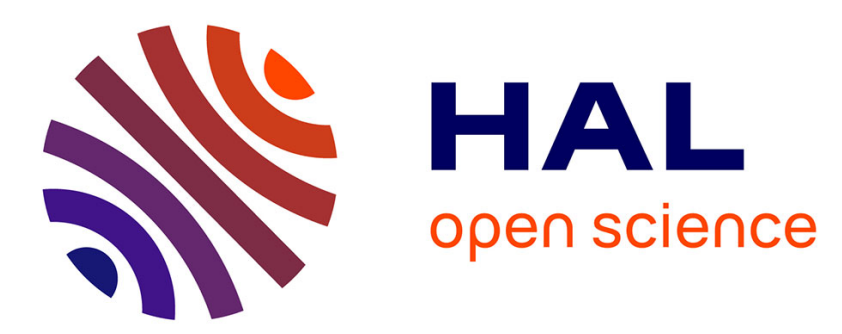

\title{
Inégalités de niveaux de vie des actifs et des retraités français: ce que nous apprend la multi-décomposition de l'indice de Gini
}

Bérangère Legendre

\section{- To cite this version:}

Bérangère Legendre. Inégalités de niveaux de vie des actifs et des retraités français: ce que nous apprend la multi-décomposition de l'indice de Gini. Revue d'Economie Politique, 2012, 5 (122), pp.727-748. hal-00951715

\section{HAL Id: hal-00951715 https://hal.science/hal-00951715}

Submitted on 25 Feb 2014

HAL is a multi-disciplinary open access archive for the deposit and dissemination of scientific research documents, whether they are published or not. The documents may come from teaching and research institutions in France or abroad, or from public or private research centers.
L'archive ouverte pluridisciplinaire HAL, est destinée au dépôt et à la diffusion de documents scientifiques de niveau recherche, publiés ou non, émanant des établissements d'enseignement et de recherche français ou étrangers, des laboratoires publics ou privés. 
Inégalités de niveaux de vie des actifs et des retraités français: ce que nous apprend la multi-décomposition de l'indice de Gini

Bérangère Legendre ${ }^{1}$

Janvier 2012

\footnotetext{
${ }^{1}$ ATER à l'Université Paris-Dauphine (LEDa-DIAL UMR225) et docteur de l'Université d'Orléans (LEO). Adresse professionnelle : Université Paris Dauphine, Place du Maréchal de Tassigny, 75775 PARIS Cedex 16 E-mail : berangere.legendre@dauphine.fr

Tel. : 0623683711
} 


\title{
Résumé
}

Le présent article propose l'analyse la plus récente, à notre connaissance, sur les inégalités entre les actifs et les retraités, et la redistribution. Nous présentons une analyse statistique approfondie des inégalités parmi les actifs, parmi les retraités, ainsi qu'entre actifs et retraités, à l'aide d'un nouvel outil de multi-décomposition des inégalités. L'outil GMR (Gini Mean Ratio) de Mussard et Terraza (2009) peut être considéré comme un outil d'aide à la décision en matière de politique économique car il fournit un panorama complet des inégalités. Le GMR met en évidence simultanément les populations concernées par les inégalités et les sources de revenus qui en sont la cause. Nous proposons donc d'étudier les apports d'une telle méthode appliquée à la problématique des inégalités entre actifs et retraités. En 2007, l'impôt sur le revenu et la taxe d'habitation acquittés par les actifs permettent de réduire l'indicateur d'inégalité totale de 5,0\% tandis que les prestations sociales qu'ils reçoivent entraînent une baisse de $1.3 \%$ de ce dernier. Les revenus des pensions de retraites expliquent une faible part des inégalités totales au regard de la proportion de retraités dans l'échantillon. Les pensions perçues par les retraités contribuent pour $7,7 \%$ des inégalités totales en 2007 , alors que les retraités représentent $19 \%$ de notre échantillon total.

Mots clés : Distribution des revenus, Pensions publiques, Fiscalité et Redistribution, Inégalité

Classification JEL : D31, H55, H20, D63

\author{
Inequalities in standards of living \\ among workers and retirees: \\ a multi-dimensional decomposition of the Gini coefficient
}

\begin{abstract}
In this paper, we apply a multidimensional decomposition of the Gini index to the active and retired populations in France. The multi-decomposition of an inequality measure allows us to put into perspective the role of social spending and taxation in reducing inequalities simultaneously among active people, among retirees, and between workers and pensioners. However, we question the extent to which this multidimensional inequality indicator is a relevant tool for the study of intergenerational inequality that can deliver conclusions regarding retirement policies in the context of an aging population.

In 2007, pensions explained a small proportion of the inequalities, compared with the share of retirees in the sample. Pensions received by retirees were responsible for $7.7 \%$ of overall inequality in 2007 whereas retirees account for $19 \%$ of our total sample. We also conclude that this inequality measure does not provide a clear picture of the inequalities between active individuals and retirees.
\end{abstract}

Key Words: Inequality, Personal Income Distribution, Public Pensions, Taxation and Redistribution.

JEL Classification: D31, H55, H20, D63 


\section{Introduction}

La redistribution organisée par l'Etat permet de garantir la cohésion et la justice sociale au sein des sociétés développées, de protéger les individus contre divers risques (maladie, vieillesse, etc.), et de solvabiliser les plus démunis en leur garantissant un revenu minimum (Ducamin, Baconnier et Briet, 2006). Le taux de pauvreté ${ }^{2}$ avant transferts sociaux en France était de $23,8 \%$ en 2009 (Eurostat). II n'atteignait plus que 12,9\% après versement des transferts sociaux. Les enjeux majeurs de la redistribution, c'est-à-dire de l'organisation des prélèvements sociaux et fiscaux, des versements de prestations sociales, contributives ou non, sont de réduire le risque de pauvreté, mais également le niveau des inégalités.

Les rapports de Ducamin, Baconnier et Briet en 1996, de Bourguignon en 1998 et du Conseil des prélèvements obligatoires en 2008 montrent que la redistribution découle, de manière générale, plus du ciblage des différentes prestations sociales que de la progressivité de l'impôt sur le revenu. Bourguignon (1998) apporte une précision supplémentaire: les instruments de redistribution sont plus les transferts sous condition de ressources parmi les personnes à faible revenu, tandis qu'il s'agit plutôt de l'impôt sur le revenu dans le haut de la distribution des revenus. En 2008, le rapport du Conseil des prélèvements obligatoires montre que l'imposition directe entraîne une redistribution des personnes en fin de carrière vers les plus jeunes et les plus âgés.

La lutte contre les inégalités étant un enjeu majeur de politique économique, et une des raisons de l'organisation de la redistribution, une bonne connaissance du niveau effectif des inégalités, des populations concernées et des leviers d'action potentiels semble indispensable. C'est pourquoi nous proposons dans cet article d'utiliser l'outil de multidécomposition des inégalités de Mussard et Terraza (2009), le GMR, et de voir dans quelles mesures il permet de renseigner sur les inégalités actifs/retraités en France. L'utilisation de l'outil "Gini Mean Ratio » (GMR) de Mussard et Terraza (2009) nous permet de mettre en lumière les contributions marginales des groupes de populations mais aussi de leurs différentes sources de revenu à l'inégalité totale.

La multi-décomposition de l'inégalité permet de constater dans quelles proportions les prestations sociales et les prélèvements fiscaux influencent le niveau d'inégalité. Mussard et Terraza (2009) avancent que le GMR permet d'identifier le meilleur ciblage en matière de réduction des inégalités. L'utilité de cette méthode en matière de politique économique n'est pas négligeable. En effet, en identifiant à la fois les sources de revenus et les échantillons d'individus à l'origine de l'inégalité, le décideur politique dispose d'un tableau de bord détaillé des inégalités sur lesquelles il peut agir. Identifier séparément les sources et les groupes de personnes constituant les inégalités ne fournit qu'une vision partielle et segmentée de la réalité. Qui cibler et avec quel outil ? Si le décideur public souhaite mener une politique ciblée de réduction des inégalités (lutte contre les inégalités dans des régions défavorisées par exemple), le GMR devrait permettre d'indiquer quel outil de politique économique utiliser.

L'information donnée par le GMR décrivant la réalité, elle s'inscrit dans la sphère de l'économie positive, par opposition à une notion normative qui impliquerait de déterminer le «bon » niveau d'inégalité. Néanmoins, si le GMR permet d'obtenir une information plus

\footnotetext{
${ }^{2}$ Au seuil de $60 \%$ du revenu médian.
} 
riche que les indicateurs classiques d'inégalités, nous nous interrogeons sur la signification exacte des résultats qu'il nous fournit pour une décomposition de l'échantillon entre individus actifs et individus retraités. Comment interpréter ces résultats statistiques dans le débat actuel sur les inégalités entre actifs et retraités ?

Dans le présent article, nous distinguons un échantillon d'actifs et de retraités. La dernière réforme du système de retraite français a fait de l'équité et de la lutte contre l'inégalité à la retraite un point important. C'est pourquoi nous proposons ici une multi-décomposition des inégalités parmi les actifs et les retraités, tout en analysant l'apport d'un tel outil dans le débat de politique économique relatif aux retraites. Dans le contexte de réforme des retraites que connaît actuellement la France, comme nombre d'autres pays de I'OCDE, établir une cartographie claire des inégalités et identifier les sources de revenus et de prélèvements mais également les groupes d'individus expliquant les inégalités pourrait s'avérer nécessaire pour adopter des mesures en ayant une connaissance précise et détaillée des mécanismes de redistribution en jeu au sein de la population. Le GMR peut-il fournir des réponses pertinentes?

La première partie de l'article est consacrée à la présentation des indicateurs d'inégalités. La méthode de multi-décomposition des inégalités y est notamment exposée. Nous présentons dans la seconde partie une application empirique de l'outil au cas des actifs et des retraités tout en nous interrogeant sur son apport dans le débat actuel sur les inégalités actifs/retraités. 


\section{Les mesures de l'inégalité}

Un certain nombre d'outils statistiques permettent de mesurer les inégalités au sein d'une population donnée. Le plus connu est l'indice de Gini (1921). Il est calculé à partir de la part cumulée de revenu des différentes tranches de populations. Une partie de la littérature sur les inégalités propose d'introduire un paramètre de sensibilité à l'inégalité au sein des mesures d'inégalités (Theil, 1967; Atkinson, 1970). L'indice de Gini le plus connu est également appelé indice de Gini standard. II correspond à un indice généralisé (Yitzakhi, 1943 ; Donaldson et Weymark, 1980) pour un paramètre d'aversion égal à 2.

L'indice de Gini (1921) standard peut être formulé de la manière suivante :

$$
G=1-\sum_{q}\left(X_{q+1}-X_{q}\right)\left(Y_{q+1}+Y_{q}\right)
$$

où $q$ représente la population classée par quantiles, $X$ la population cumulée et $Y$ le revenu cumulé. Le coefficient de Gini peut également être formulé en faisant apparaître les écarts de revenus absolus entre individus pris deux à deux :

$$
\Leftrightarrow G=\sum_{i=1}^{n} \sum_{r=1}^{n} \frac{\left|x_{i}-x_{r}\right|}{2 N^{2} \bar{x}}
$$

Où $x_{i}$ et $x_{r}$ sont les revenus des individus $i$ et $r$ dans une population de $\mathrm{N}$ individus. $\bar{x}$ représente le revenu moyen.

L'indice de Gini généralisé a été popularisé par les travaux de Donaldson et Weymark (1980), et de Yitzhaki (1983) :

$$
\begin{gathered}
I_{\rho}=\frac{v-\zeta_{\rho}}{v} \\
\operatorname{avec} \zeta_{\rho}=\sum_{l=1}^{L}\left[\frac{\left(R_{t}\right)^{\rho}-\left(R_{t+1}\right)^{\rho}}{\left(R_{1}\right)^{\rho}}\right] y_{l} \\
\text { et } R_{l}=\sum_{i=l}^{L} \omega_{i}
\end{gathered}
$$

Où $v$ représente la moyenne des revenus, $\omega_{l}$ et $y_{l}$ le poids et le niveau de revenu de l'individu, et $\rho$ le paramètre d'aversion à l'inégalité.

Les formules d'entropie généralisée ont été développées par Theil puis reprises par Shorrocks (1980) qui voulait appliquer une mesure de désordre dans un système thermodynamique à la mesure des inégalités entre individus. Dans les formules d'entropie généralisée, un paramètre $\alpha$ représente le poids donné aux distances entre les revenus en différents points de la distribution. Plus il est bas, plus l'indicateur d'inégalité est sensible 
dans la région inférieure de la distribution, et inversement. La valeur 0 indique l'égalité parfaite au sein de l'échantillon considéré. Lorsque l'indicateur prend la valeur 0.5 , cela indique que $74 \%$ des individus possèdent $26 \%$ des ressources, tandis que $26 \%$ des individus en possèdent $74 \%$.

L'indice de Theil (1967) est calculé grâce à l'équation d'entropie généralisée $G E_{\alpha}$ (Shorrocks, 1980) :

$$
\begin{gathered}
G E_{\lim \alpha \rightarrow 1}=\frac{1}{\alpha(\alpha-1)}\left[\frac{1}{N} \sum_{i=1}^{N}\left(\frac{y_{i}}{\bar{y}}\right)^{\alpha}-1\right] \\
\alpha<1
\end{gathered}
$$

$\mathrm{N}$ représentant la taille de la population, $y_{i}$ le revenu de l'individu $i, \bar{y}$ le revenu moyen. Le paramètre $\alpha$ représente le poids donné aux distances entre les revenus en différents points de la distribution (Sautory, 1996). Les valeurs les plus communément utilisées sont 0, 1 et 2 . Si $\alpha=0$, un poids plus important est attribué aux distances entre revenus du bas de la distribution. L'indicateur devient plus sensible aux changements dans le bas de la distribution. Si $\alpha=1$, un même poids est attribué aux distances sur toute la distribution. Enfin, si $\alpha=2$, l'indicateur donne proportionnellement plus de poids aux distances entre revenus du haut de la distribution.

L'indicateur d'Atkinson fait quant à lui appel à une norme de prise en compte des inégalités. Cette norme $\varepsilon$ permet à l'image de l'indicateur de Theil, d'accorder plus ou moins d'importance aux inégalités selon que l'on se trouve dans le haut ou dans le bas de la distribution des revenus. Plus $\varepsilon$ est élevé, plus la société est sensible à l'inégalité (Litchfield, 1999). L'indicateur d'Atkinson représente la perte de revenu que seraient prêts à accepter les individus pour que la distribution du revenu soit égalitaire: un indicateur de $x \%$ indique que la population accepterait de céder $x \%$ de ses ressources en échange d'une distribution égalitaire.

L'indicateur d'Atkinson (1970), $A_{\varepsilon}$, se formule comme suit :

$$
\begin{gathered}
A_{\varepsilon}=1-\left[\frac{1}{N} \sum_{i=1}^{N}\left(\frac{y_{i}}{\bar{y}}\right)^{1-\varepsilon}\right]^{\frac{1}{(1-\varepsilon)}} \\
\varepsilon \in] 0,+\infty[
\end{gathered}
$$

Les valeurs $0.5,1$ et 2 sont couramment utilisées, elles indiquent une sensibilité croissante à l'inégalité.

On montre aussi que :

$$
A_{\alpha}=1-\left[\frac{1}{N} \sum_{i=1}^{N}\left(\frac{y_{i}}{\bar{y}}\right)^{2-\alpha}\right]^{\frac{1}{(2-\alpha)}}
$$


Quand $\varepsilon=\alpha-1$ pour $\alpha<1$, où $\alpha$ est le paramètre d'aversion à l'inégalité dans la formule d'entropie. Les mesures d'entropie généralisée sont alors équivalentes aux mesures d'Atkinson (Cowell, 1995). $n$ est la taille de la population, $y_{i}$ le revenu de l'individu $i$, et $\bar{y}$ le revenu moyen.

Les critères de mesure de l'inégalité dépendent des axiomes qui les caractérisent. Les mesures issues de la notion d'entropie développée par Theil respectent l'axiome de décomposition additive ${ }^{3}$, il est donc possible de décomposer l'inégalité totale en inégalités entre sous-groupes (en fonction du genre, de l'activité, de la dispersion géographique, etc.). Cette décomposabilité permet de prendre en compte l'hétérogénéité existant entre agents de groupes différents et de mettre en évidence les contributions des inégalités intergroupes et intragroupes à l'inégalité totale.

Shorrocks (1980), Lerman et Yitzhaki (1985) ont mis en évidence un autre type de décomposition d'indice d'inégalité : la décomposition par source de revenu. Ainsi, ce type de méthode permet de mettre en évidence la contribution des revenus d'activité, des revenus du capital encore des différentes prestations sociales dans l'inégalité totale de revenu disponible.

Les uns ou les autres indicateurs peuvent être utilisés en fonction du contexte, ou encore selon le but recherché : mettre en lumière la sensibilité d'un groupe à l'inégalité envers les plus pauvres, calculer les contributions marginales de différentes sources de revenus ou de richesse à l'inégalité totale, ou encore les contributions de différents sous-échantillons, au sein d'une population mère.

Mussard et Terraza (2009) proposent quant à eux de regrouper dans une même mesure les contributions de différents groupes d'individus, exclusifs et exhaustifs, et différentes sources de revenus à l'inégalité totale. Ils établissent qu'il existe une mesure de l'inégalité, décomposable en indicateurs secondaires permettant de calculer simultanément :

- la contribution de chaque sous-groupe de population à l'inégalité totale,

- la contribution de chaque source de revenu,

- $\quad$ et la contribution croisée de chaque groupe et chaque source.

La mutli-décomposition permet d'identifier les couples d'inégalités "source de revenu/inégalité intragroupe" et "source de revenu/inégalité intergroupe" (Cf. tableau 1). Les auteurs proposent la formule d'un indicateur appelé rapport moyen de Gini (GMR) et fournissent une application de cet indicateur d'inégalité sur données italiennes, ce qui leur permet de décomposer simultanément l'inégalité globale en Italie :

- en inégalité issue des différentes sources de revenu (salaires, primes, pensions, transferts, etc.),

- en inégalité géographique (Nord, Centre, Sud)

- en inégalité croisée source/région.

\footnotetext{
${ }^{3}$ La décomposabilité additive implique que l'inégalité totale est la somme de deux composantes: I'inégalité intra-groupes, et l'inégalité inter-groupe (Shorrocks, 1980 ; Mussard et Terraza, 2009).
} 
L'indice GMR correspond à un indicateur de Gini généralisé où le paramètre d'aversion serait égal à 1 . Il respecte un principe de transfert plus large que le principe de transfert de Pigou Dalton: il respecte le principe de concentration d'Ebert (2010). La propriété de transfert de Pigou-Dalton est essentielle à un indicateur d'inégalité : toute redistribution d'un individu riche vers un individu moins riche, telle que l'ordre des deux individus dans la distribution des revenus ne change pas, devrait diminuer l'inégalité. Le principe de concentration d'Ebert permet d'analyser non plus l'impact d'un transfert entre deux individus, mais entre plusieurs échantillons, et donc de tester une gamme plus large de politiques de transfert.

La particularité du GMR est de se rapprocher de ce qu'Ebert (2010) a nommé " indices faiblement décomposables " par opposition aux indices " additivement décomposables " comme l'indice de Theil. Chacune des deux classes d'indices décomposables intègre un indicateur d'inégalité intragroupe et indicateur d'inégalité intergroupe. La composante intragroupe équivaut dans les deux cas à une somme pondérée des inégalités de revenu au sein des groupes. Mais la mesure de l'inégalité intergroupe diffère. Dans le cas des indicateurs additivement décomposables, l'inégalité intergroupe fait intervenir une moyenne de revenus ou un revenu représentatif du groupe. Les indicateurs dits "faiblement " décomposables intègrent un indice intergroupe plus développé, qui permet de comparer chaque revenu d'un groupe à chaque revenu d'un autre groupe. Autrement dit l'inégalité intergroupe est mesurée en comparant directement toutes les paires de revenus possibles entre deux groupes ${ }^{4}$. Cela permet donc de tenir compte de toutes les distributions de revenus au sein de chaque groupe plutôt que de n'intégrer qu'une valeur représentative pour chacun des groupes. Ainsi, lorsque deux individus $i$ et $j$ de la population sont tirés au sort $^{5},(1-G M R)$ donne la proportion de revenu $x_{i}$ détenue par l'individu $j$.

\footnotetext{
${ }^{4}$ La multi-décomposition permet de surmonter le problème de « no bridge » régulièrement rencontré dans la littérature sur les inégalités, c'est-à-dire l'utilisation exclusive soit de mesures décomposables en sous groupes, soit de mesures décomposables en sources de revenus. Mussard et Terraza (2009) montrent qu'en mobilisant la famille des mesures d'inégalité décomposables en sous-populations par les comparaisons par paires, il est possible de surmonter cette impasse de « no bridge ». Pour plus de détails, voir Mussard et Terraza (2009).

${ }^{5}$ Il s'agit d'un tirage au sort avec remise et avec $x_{i}>x_{j}$
} 
Tableau 1 Contributions aux inégalités fournies par le GMR

\begin{tabular}{|c|c|c|c|c|c|}
\hline & $\begin{array}{c}\text { Source de revenu } \\
1\end{array}$ & $\begin{array}{c}\text { Source de } \\
\text { revenu } 2\end{array}$ & ... & $\begin{array}{c}\text { Source } \\
n\end{array}$ & Revenu Total \\
\hline $\begin{array}{c}\text { Sous- } \\
\text { échantillon } 1\end{array}$ & $\begin{array}{l}\text { Inégalité due à la } \\
\text { source } 1 \text { perçue } \\
\text { par le sous- } \\
\text { échantillon } 1\end{array}$ & & & & $\begin{array}{c}\text { Inégalité } \\
\text { marginale } \\
\text { due au sous- } \\
\text { échantillon } 1\end{array}$ \\
\hline $\begin{array}{c}\text { Sous- } \\
\text { échantillon } 2\end{array}$ & & & & & $\begin{array}{c}\text { +inégalité } \\
\text { maginale due } \\
\text { au sous- } \\
\text { échantillon } 2\end{array}$ \\
\hline$\ldots$ & & & & & $+\ldots$ \\
\hline $\begin{array}{c}\text { Sous- } \\
\text { échantillon k }\end{array}$ & & & & & $+\ldots$ \\
\hline $\begin{array}{c}\text { Croisement } \\
\text { sous- } \\
\text { échantillon } 1 \\
\text { - sous } \\
\text { échantillon } 2\end{array}$ & & & & & $+\ldots$ \\
\hline$\cdots$ & & & & & $+\ldots$ \\
\hline $\begin{array}{c}\text { Echantillon } \\
\text { total }\end{array}$ & $\begin{array}{c}\text { Inégalité } \\
\text { marginale due à la } \\
\text { source } 1\end{array}$ & $\begin{array}{c}\text { + Inégalité } \\
\text { marginale } \\
\text { due à la } \\
\text { source } 2\end{array}$ & $+\ldots$ & $+\ldots$ & $=\mathrm{GMR}$ \\
\hline
\end{tabular}

Source : Mussard et Terraza, 2009

L'expression mathématique du GMR a donc deux composantes: la première partie de l'expression mesure l'inégalité intra-groupe, la seconde, l'inégalité intergroupe. Ainsi, la formule de Mussard et Terraza (2009) permet de comparer les revenus deux à deux au sein des groupes, puis de répéter cette opération au sein de chaque sous-échantillon. De la même manière, sont comparés deux à deux les revenus des individus appartenant cette fois à des sous-échantillons différents. L'inégalité totale calculée par le GMR est indépendante des groupes d'individus choisis pour la décomposition. Les groupes choisis sont disjoints.

Mussard et Terraza montrent qu'il est possible de rapprocher les formes analytiques des indicateurs d'inégalités d'une part décomposables en inégalités issues des sources de revenus, et d'autre part décomposables en inégalités issues de différents sous échantillons. Ce rapprochement permet de définir l'indicateur multi-décomposable. En reprenant les notations des auteurs, les inégalités de revenu mesurées sur un vecteur $x$ se décomposent en inégalités intragroupes et intergroupes : ainsi l'équation (1) se compose d'une première partie $\left(G M R_{j j}\right)$ faisant état des inégalités entre les $n_{j}$ individus d'un sous-échantillon $j$, et d'une seconde partie $\left(G M R_{j h}\right)$ mettant en lumière les inégalités entre les $n_{j}$ et $n_{h}$ individus des groupes $j$ et $h$. La population mère se compose de $k$ sous-échantillons et $n$ individus.

$$
G M R(x)=\sum_{j=1}^{k} z_{j} G M R_{j j}+2 \sum_{j=2}^{k} \sum_{h=1}^{j-1} z_{j h} G M R_{j h}
$$




$$
\Leftrightarrow G M R(x)=\sum_{j=1}^{k} z_{j} G M R_{j j}+\sum_{\substack{j, h=1 \\ j \neq h}}^{k} z_{j h} G M R_{j h}
$$

Où $z_{j}$ et $z_{j h}$ sont deux indicateurs de proportion des $n_{j}$ et $n_{h}$ individus issus des $k$ souséchantillons par rapport à la population mère de $n$ individus :

$$
\begin{gathered}
z_{j}=\frac{n_{j}^{2}}{n^{2}} \\
z_{j h}=\frac{n_{j} n_{h}}{n^{2}}
\end{gathered}
$$

$G M R_{j j}$, la contribution de l'inégalité intragroupe mesurant la dispersion des revenus $\left(x_{i j}\right.$ et $x_{r j}$ ) entre les individus $i$ et $r$ du groupe $j$ pris deux à deux, s'écrit :

$$
G M R_{j j}=\sum_{i=1}^{n_{j}} \sum_{r=1}^{n_{j}} \frac{\left|x_{i j}-x_{r j}\right|}{n_{j}^{2} \max \left(x_{i j}, x_{r j}\right)}
$$

Tandis que $G M R_{j h}$, la contribution de l'inégalité intergroupe mesurant les écarts de revenu entre les individus du sous-échantillon $j$ d'une part et du sous-échantillon $h$ d'autre part, peut être formulée comme suit :

$$
G M R_{j h}=\sum_{i=1}^{n_{j}} \sum_{r=1}^{n_{h}} \frac{\left|x_{i j}-x_{r h}\right|}{n_{j} n_{h} \max \left(x_{i j}, x_{r h}\right)}
$$

Les équations (1) à (3) nous fournissent les contributions des différents groupes de la population mère à l'inégalité totale. A cela doit être ajoutée la décomposition en différentes sources de revenus. Le vecteur des revenus $x$ se décompose en effet en $q$ sources indicées $l$.

Pour chaque individu $i$ du groupe $j$, on a :

$$
x_{i j}=\sum_{l=1}^{q} x_{i j}^{l}
$$

Alors, l'équation (1) peut être réécrite, de manière à afficher d'une part les inégalités intra et inter-groupe, et d'autre part les contributions des différentes sources de revenu $x^{l}$ à l'inégalité totale :

$$
\operatorname{GMR}(x)=\sum_{l=1}^{q} \sum_{j=1}^{k} \sum_{i=1}^{n_{j}} \sum_{r=1}^{n_{j}} z_{j} \frac{x_{i j}^{l}+x_{r j}^{l}-2 \min \left(x_{i j}^{l}, x_{r j}^{l}\right)}{n_{j}^{2} \max \left(x_{i j}, x_{r j}\right)}+2 \sum_{l=1}^{q} \sum_{j=2}^{k} \sum_{h=1}^{j-1} \sum_{i=1}^{n_{j}} \sum_{r=1}^{n_{h}} z_{j h} \frac{x_{i j}^{l}+x_{r h}^{l}-2 \min \left(x_{i j}^{l}, x_{r h}^{l}\right)}{n_{j} n_{h} \max \left(x_{i j}, x_{r h}\right)}
$$




$$
\Leftrightarrow G M R(x)=\sum_{l=1}^{q} \sum_{j=1}^{k} \sum_{i=1}^{n_{j}} \sum_{r=1}^{n_{j}} z_{j} \frac{x_{i j}^{l}+x_{r j}^{l}-2 x_{j, i r}^{* l}}{n_{j}^{2} \max \left(x_{i j,} x_{r j}\right)}+2 \sum_{l=1}^{q} \sum_{j=2}^{k} \sum_{h=1}^{j-1} \sum_{i=1}^{n_{j}} \sum_{r=1}^{n_{h}} z_{j h} \frac{x_{i j}^{l}+x_{r h}^{l}-2 x_{j h, i r}^{* l}}{n_{j} n_{h} \max \left(x_{i j} x_{r h}\right)}
$$

Où $x_{j, i r}^{* l}$ et respectivement $x_{j h, i r}^{* l}$, donnent la $l^{\text {ème }}$ source de revenu: elles prennent respectivement la valeur du minimum des revenus $x_{i j}$ et $x_{r j}$, ou de $x_{j h}$ et $x_{i r}$ et ce, pour chaque couple de revenu potentiel au sein du sous-échantillon $j$, ou entre les échantillons $j$ et $h$ (Voir annexe A pour les détails de calcul supplémentaires).

Nous appliquons par la suite l'indicateur GMR sur données françaises de 2007, en distinguant simultanément les sources de revenus disponibles et les actifs des retraités.

\section{Multi-décomposition de l’inégalité entre actifs et retraités en France}

Les données du dispositif français SRCV (Statistiques sur les ressources et les conditions de vie) inclus dans l'enquête européenne EU-SILC (European Survey on Income and Living Conditions) et réalisé par l'Institut National de la Statistique et des Etudes Economiques (INSEE) sont mobilisées pour proposer une décomposition sur deux axes de l'inégalité entre actifs et retraités.

L'enquête européenne EU-SILC a été menée pour la première fois en 2004. Elle s'articule depuis autour de deux axes : un axe longitudinal sous forme de panel, mais également un axe transversal, à l'image d'une enquête traditionnelle. Le dispositif français SRCV (Statistiques sur les ressources et les conditions de vie) traite des questions relatives à la pauvreté et aux conditions de vie des individus et des ménages. II est composé d'une table " ménages » et d'une table " individus ». Les membres des ménages sont répertoriés au sein de la table individus, et les ménages sont constitués sur la base d'un critère de "communauté de budget ". Le SRCV renseigne notamment sur les prestations et les différents prélèvements fiscaux et sociaux supportés par les ménages, ainsi que les différentes prestations sociales. Afin de mieux comprendre l'évolution des conditions de vie des répondants, les règlements européens prévoient de suivre les ménages du panel sur au moins quatre années consécutives. La France a choisi de mettre en place un suivi plus long, de neuf ans.

Nous utilisons une approche en termes de niveaux de vie. Ce dernier est assimilé au revenu disponible du ménage, en tenant compte de sa composition. L'indicateur du rapport moyen de Gini ou GMR est donc calculé en distinguant les différentes composantes du revenu disponible perçu au sein du ménage, puis imputé individuellement à ses membres. 
Nous distinguons notamment les prélèvements fiscaux au sein du revenu disponible, ce qui exclut les prélèvements sociaux de notre revenu décomposé dans la mesure où ceux-ci sont prélevés en amont du revenu net (Cf. schéma 1 en annexe $B$ ).

La détermination du revenu disponible fait appel à des variables individuelles, telles les rémunérations salariales, et des variables propres au ménage, telles les prestations familiales ou encore les prélèvements fiscaux ${ }^{6}$. L'approche ménage a l'avantage de mieux refléter le niveau de vie des individus. Dès lors, nous nous intéressons aux inégalités de niveau de vie. Notre approche consiste ici à recréer pour chaque source de revenu ou de prélèvement son montant à l'échelon du ménage puis de l'individu au sein de chaque ménage, en tenant compte des phénomènes d'échelle qui interviennent alors.

Deux sous-échantillons sont distingués : les actifs, occupés ou inoccupés, et les retraités. Le sous-échantillon de retraités inclut les personnes ayant liquidé leurs droits à la retraite, les personnes en pré-retraite et les personnes ayant cessé leur activité. Enfin, les actifs comprennent les personnes à temps plein, à temps partiel et les chômeurs. Les étudiants et lycéens ont été ôtés de l'échantillon.

Après avoir calculé le revenu disponible au sein de chaque ménage, nous imputons à chacun de ses membres un revenu par unité de consommation (UC) : le revenu est divisé par le nombre d'unités de consommation au sein du ménage. L'échelle d'équivalence dite de I'OCDE (Organisation de Coopération et de Développement Économiques) est utilisée : une unité de consommation est attribuée au premier membre du ménage, puis 0,5 aux autres membres âgés de plus de 14 ans, et 0,3 aux enfants de moins de 14 ans. L'approche en termes de niveaux de vie permet d'éviter de considérer par exemple deux membres d'un même ménage avec des revenus parfois très différents indépendamment l'un de l'autre : au lieu de considérer fictivement une personne qui serait, du fait de sa source personnelle de revenu, sous le seuil de pauvreté et une autre dont le revenu serait très élevé, nous imputons à chacun un revenu équivalant à leur niveau de vie effectif au sein du ménage, compte tenu du critère de communauté de budget.

Le Conseil d'orientation des retraites à partir des données de l'Insee constate en 2009 que " les indicateurs comparant le niveau de vie des retraités à celui des actifs sont proches de la parité ». En 2007, les données du SRCV confirment ce constat.

Tableau 2 Niveaux de vie et rapports interquantiles en $2007^{7}$

\begin{tabular}{|c|c|c|c|c|}
\cline { 2 - 5 } \multicolumn{1}{c|}{} & \multicolumn{2}{c|}{$\begin{array}{c}\text { Montant moyen par } \\
\text { UC }\end{array}$} & \multicolumn{2}{c|}{ D9/D1 } \\
\cline { 2 - 5 } & Actifs & Retraités & Actifs & Retraités \\
\hline $\begin{array}{c}\text { Revenu } \\
\text { disponible }\end{array}$ & 22298 & 22363 & 3,5 & 3,8 \\
\hline $\begin{array}{c}\text { Pension } \\
\text { Revenu } \\
\text { d'activité }\end{array}$ & 15362 & 15770 & - & 4,5 \\
\hline
\end{tabular}

\footnotetext{
${ }^{6}$ Le détail du calcul de la variable de revenu disponible est expliqué en annexe $B$.

${ }^{7}$ Les années mentionnées sont les années d'enquête. Les revenus sont donc les revenus de l'année précédant l'enquête.
} 
Bien que les jeunes retraités aient connu des aléas de carrières plus fréquents que leur aînés (Briard et al., 2009), et que les conditions de départ en retraite se durcissent, leur niveau de vie s'est aussi élevé. La pension de retraite est certes indexée sur l'évolution des prix, mais l'effet noria ${ }^{8}$ ne peut être négligé : les générations de retraités d'aujourd'hui sont plus qualifiées que par le passé. L'effet de noria est par ailleurs beaucoup plus marqué parmi les femmes que parmi les hommes (Andrieux et Chantel, 2011). Ainsi, Burricand et Deloffre (2007) notent que la croissance observée du niveau moyen des pensions entre 2000 et 2004 était due en grande partie au renouvellement des retraités. Andrieux et Chantel (2011) offrent le même constat pour la période 2004-2008. Sur les deux périodes, deux phénomènes renforcent l'effet renouvellement des générations: d'une part les retraités entrants percevaient des revenus d'activité supérieurs à leurs aînés, d'autre part, les retraités sortants percevaient également des pensions plus faibles. Burricand et Deloffre expliquent notamment que les jeunes retraités du début des années 2000 avaient bénéficié de la période de forte croissance du pouvoir d'achat entre 1945 et 1975. Les retraités décédés au début des années 2000 percevaient par ailleurs des avantages principaux de droit direct inférieurs de 10\% en moyenne aux retraités en vie en 2000 et en 2004.

Si l'on considère uniquement les pensions pour les retraités et les revenus d'activité pour les actifs, on s'aperçoit que ces derniers sont plus élevés. En revanche, les revenus issus des pensions sont distribués de manière moins inégale que les revenus d'activité : le rapport interdécile des pensions est de 4.5 en 2007, tandis que celui des revenus d'activité est de 5.

Les revenus des retraités sont distribués de manière légèrement plus inégalitaire que ceux des actifs. Ces rapports interdéciles sont moins élevés que les rapports interdéciles calculés à l'aide des pensions et des revenus d'activité, pour les retraités et les actifs, dans la mesure où ils prennent en compte la redistribution opérée à l'aide des différentes prestations d'assistance sociale et des prélèvements fiscaux.

Les revenus issus de l'activité professionnelle (salaires et revenus des indépendants) représentent environ $93 \%$ du revenu disponible par UC des actifs. Cette source de revenu représente environ $15 \%$ du revenu disponible par UC des retraités. Les pensions de retraite équivalent à environ $88 \%$ du revenu disponible des retraités, et 3 à $4 \%$ du revenu disponible des actifs ${ }^{9}$.

Les revenus des capitaux représentent une proportion plus importante du revenu disponible des retraités que du revenu disponible des actifs. Les revenus issus des capitaux mobiliers (intérêts, dividendes) s'élèvent en moyenne à hauteur de $2 \%$ du revenu disponible par unité de consommation des actifs en 2007. Pour les retraités, ces revenus équivalent à environ $5 \%$ du revenu disponible.

\footnotetext{
${ }^{8}$ L'effet noria mesure, au sein d'une entreprise/organisation, l'évolution de masse salariale due à la différence de rémunération entre les salariés sortants et les salariés entrants.

${ }^{9}$ Il peut paraître surprenant de parler du revenu issu d'une pension de retraite pour les personnes actives, mais rappelons que nous considérons ici un revenu par unité de consommation, parfois aussi appelé revenu équivalent. Autrement dit, ce revenu tient compte du niveau de vie du ménage et donc de toutes les sources de revenu au sein de celui ci, moyennant sa taille.
} 
En dépit de la fiscalité préférentielle accordée aux retraités ${ }^{10}$, l'impôt sur le revenu et la taxe d'habitation représentent un poids plus important dans le revenu disponible des retraités, que dans celui des actifs : $8,49 \%$ pour les premiers, contre $7.42 \%$ pour les seconds.

Enfin, l'impôt de solidarité sur la fortune représente un poids faible relativement au revenu disponible des actifs et des retraités (moins de $1 \%$ ). Néanmoins, ce prélèvement fiscal représente un poids légèrement supérieur relativement revenu disponible des retraités: $0,50 \%, 0,14 \%$ pour les actifs.

\section{Orientation redistributive vers les actifs}

En 2007, l'indicateur GMR s'élève à 0.36 (Cf. tableau 3). Ainsi un individu $j$ pris au hasard perçoit en moyenne $64 \%$ du revenu d'un individu $i$.

47.1\% des inégalités exprimées par le GMR sont imputables aux seuls actifs, $43.1 \%$ aux inégalités entre actifs et retraités. La forte contribution croisée s'explique par la structure même du GMR : la comparaison par paire permet certes de prendre en compte toute la distribution du revenu, mais conduit également à comparer des actifs très riches avec des retraités très pauvres, et inversement. Cette forte composante croisée de l'inégalité totale doit donc être observée avec précaution : il peut s'avérer utile de lutter contre les inégalités entre les actifs et les retraités, mais cela passerait en partie par une atténuation des très forts écarts existants entre des minorités très riches et le reste de la population, aussi bien parmi les actifs que parmi les retraités. Il s'agit là, à notre sens, d'une limite du GMR. Appliqué à la problématique des inégalités actifs/retraités, la forte composante intergroupe laisse supposer qu'en réduisant les inégalités entre les actifs et les retraités, le décideur politique réduirait l'inégalité totale dans la population. Or les niveaux de vie des deux populations sont aujourd'hui, en moyenne, comparables.

Parmi les retraités, les valeurs très élevées de certains revenus disponibles ne trouvent pas leur fondement principal dans la pension de retraite fournie par le régime par répartition, mais plutôt dans la constitution de patrimoines importants sur le cycle de vie, voire le plus souvent sur plusieurs générations. On peut donc douter de l'utilité d'une stricte politique des retraites à ce niveau.

Seuls 9,7\% sont imputables aux inégalités intra-retraités (Cf. tableau 3). Bien que les paires de retraités représentent $19 \%$ des paires observées parmi nos échantillons en 2007 (Cf. tableau 4 en annexe $B)^{11}$, les inégalités parmi eux expliquent moins de $10 \%$ des inégalités totales. Les retraités semblent donc générer une sous contribution à l'inégalité totale bien qu'ils présentent un rapport inter décile supérieur à celui des actifs (Cf. tableau 2). En effet, les retraités les plus riches perçoivent en moyenne des revenus plus élevés que les actifs les plus riches.

\footnotetext{
${ }^{10}$ Les pensionnés bénéficient notamment d'un abattement de $10 \%$ sur les pensions au titre de l'impôt sur le revenu. Certains cas de figure prévoient également la non imposition, l'exonération, le dégrèvement ou le plafonnement de la taxe d'habitation pour les retraités.

${ }^{11}$ Les retraités représentent $44 \%$ de l'échantillon total, mais les paires « retraités-retraités » comparées par le GMR ne représentent que $44 \% * 44 \%$, soit $19 \%$ du total.
} 
Figure 1 Contributions marginales des sous-échantillons, année 2007

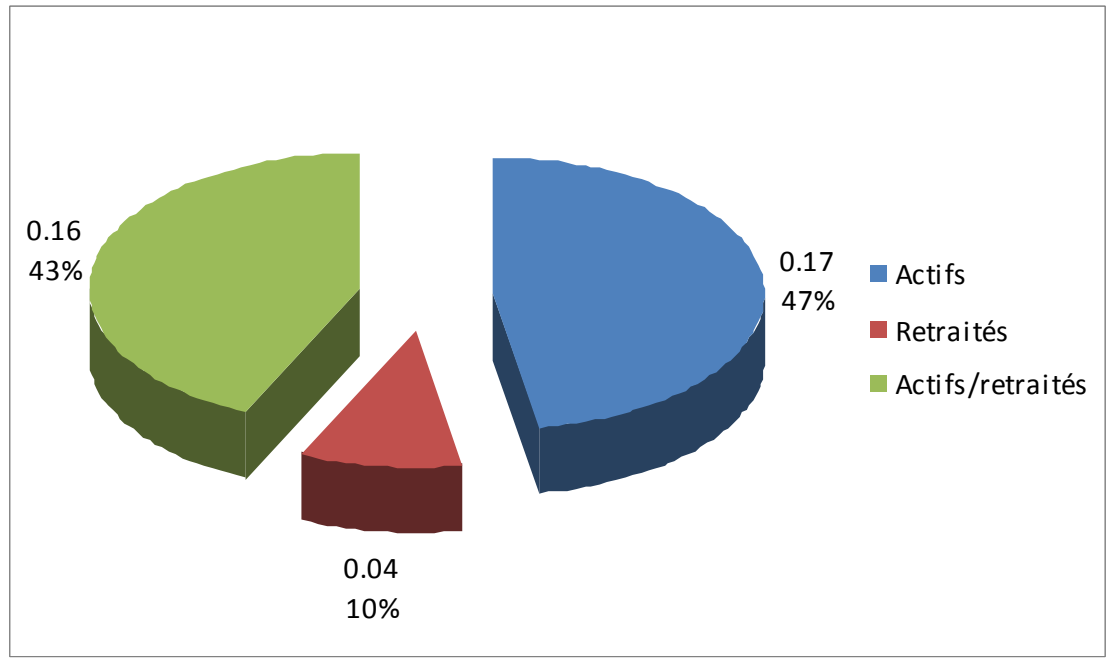

Lecture : 46\% des inégalités sont attribuables aux inégalités parmi les actifs. Source : SRCV, calculs de l'auteur.

La précision qu'offre la décomposition de l'indice d'inégalité permet de montrer que la participation des retraités à l'inégalité totale est due en majeure partie aux inégalités de pension de retraite. En 2007, cette source de revenu contribue pour 20,7\%, soit un indicateur de contribution marginale de 0,07 . Cela se vérifie également pour le croisement de cette source de revenu et du sous-échantillon des retraités : la part de l'inégalité totale imputable à la perception de pensions de vieillesse par les retraités s'élève à 7,7 \% en 2007 . Les revenus issus de l'activité professionnelle perçus par les ménages avec au moins un retraité représentent $2,2 \%$ du GMR.

Figure 2 Contributions marginales des principales sources constitutives du revenu disponible, année 2007

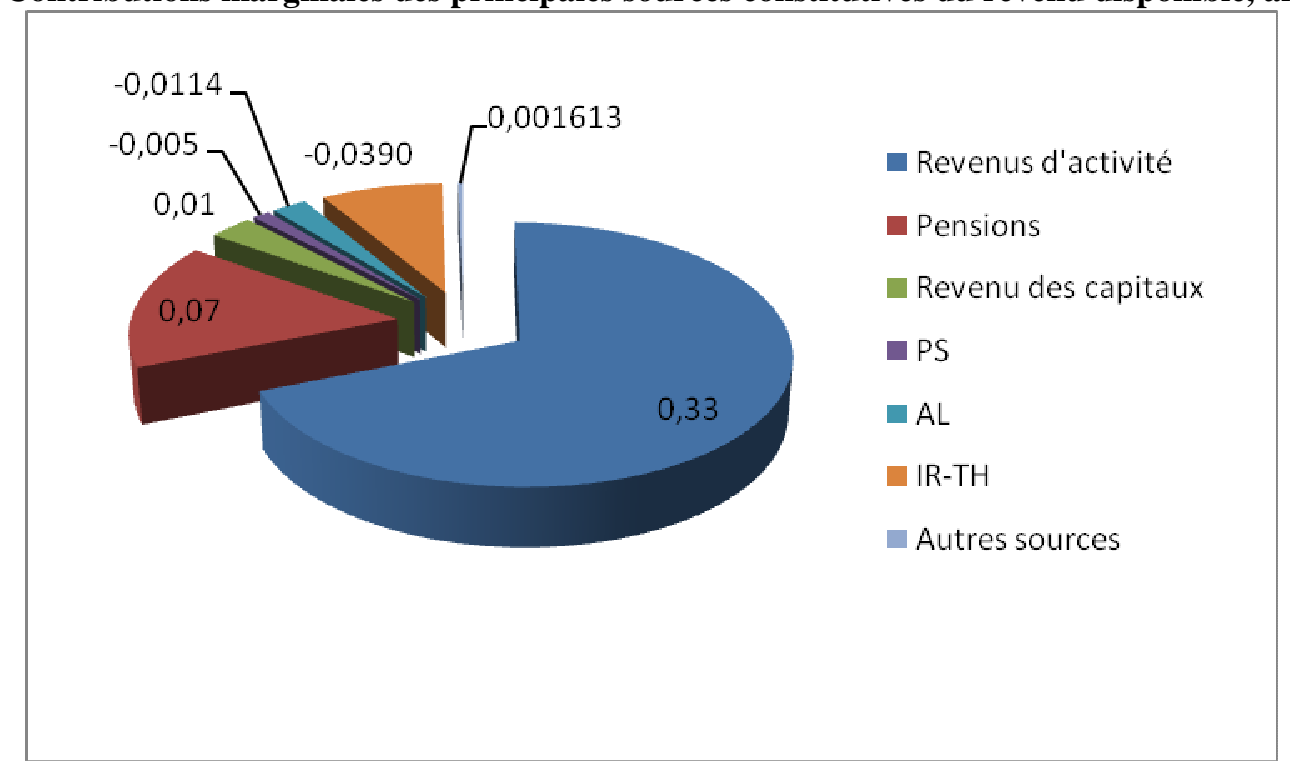

Légende : PS=prestations sociales ; $A L=$ allocations logement ; IR-TH=impôt sur le revenu et taxe d'habitation, $R D=$ revenu disponible

Lecture : La contribution marginale des revenus d'activité aux inégalités totale est de 0.33, soit 90\% du total (Cf. tableau 3).

Source : SRCV, calculs de l'auteur. 
Figure 3 Distribution des contributions marginales des principales sources de revenu parmi les sous échantillons, année 2007

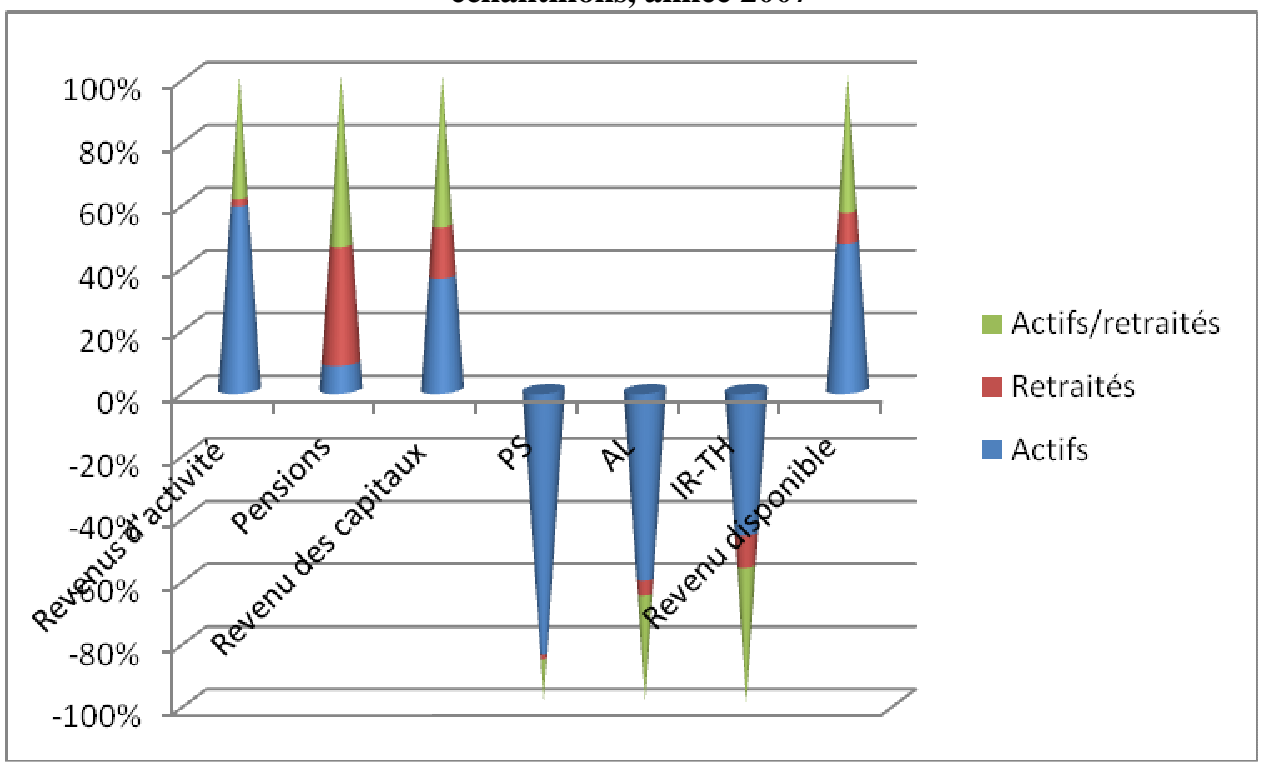

Légende : $P S=$ prestations sociales ; $A L=$ allocations logement ; IR-TH=impôt sur le revenu et taxe d'habitation, $R D=$ revenu disponible

Lecture : Plus de la moitié(0.19/0.33=58\%, cf. tableau 3 ) de la contribution marginale des revenus d'activité concerne les inégalités parmi les actifs.

Source : SRCV, calculs de l'auteur.

Les différentes prestations non contributives permettent de diminuer les inégalités, que ce soit parmi les actifs, les retraités ou encore entre les actifs et les retraités. C'est plus particulièrement le cas de l'aide au logement et des prestations familiales, qui, en 2007, diminuaient l'indicateur d'inégalité respectivement de $3.2 \%$ et 1,5\%.

Le détail du résultat par sous-échantillon nous apprend également que ces aides permettent en premier lieu de diminuer les inégalités parmi les actifs. $85 \%$ de la réduction d'inégalité permise par les prestations sociales concernent ces derniers (Cf. figure 3 ). L'aide au logement, en prenant en charge une partie des dépenses de logement des ménages, qu'il s'agisse d'une partie de loyer ou de remboursement d'emprunt, réduit les inégalités de niveau de vie entre les individus. Les prestations logements permettent une réduction des inégalités moindre parmi les retraités dans la mesure où passé un certain âge, les individus sont plus couramment propriétaires de leur résidence principale, et qui plus est, en ont terminé le remboursement. A titre d'exemple, en 2006, 12,6\% des ménages de moins de 30 ans sont propriétaires de leur résidence principale, contre $72,1 \%$ pour les ménages de 65 ans ou plus. Par ailleurs, parmi ces derniers, 70,2 \% n'ont plus d'emprunt en cours (Enquête Logement, Insee).

Si la taxe foncière ne contribue pas à réduire les inégalités, l'impôt sur le revenu, la taxe d'habitation et l'impôt de solidarité sur la fortune (ISF) le permettent. La contribution de I'ISF à la réduction des inégalités est marginale : elle représente environ $-0,1 \%$ à $-0,2 \%$ de I'inégalité totale (Cf. Tableau 3), et concerne principalement les inégalités entre actifs et retraités. L'impôt sur le revenu et la taxe d'habitation représentent quant à eux un facteur important de baisse des inégalités, plus encore que les prestations non contributives. Ils permettent de diminuer l'indicateur d'inégalité totale de 10,8\% en 2007. Cela concerne 
principalement le groupe des actifs, mais aussi les inégalités entre actifs et retraités. L'impôt sur le revenu parmi les actifs permet de diminuer l'inégalité totale de 5,0\%, contre seulement $1,1 \%$ parmi les retraités. La conjugaison de la progressivité de l'impôt sur le revenu et de la fiscalité préférentielle propre aux retraités semble donc efficiente pour éviter qu'un écart trop important de niveau de vie ne se forme entre les actifs et les retraités, mais également entre actifs de productivités différentes percevant des revenus du travail inégalitaires. Nous mentionnerons à titre d'exemple l'abattement de $10 \%$ accordé aux pensions, les majorations de quotient familial ${ }^{12}$, ou encore les cas d'exonération ou de dégrèvement de la taxe $d$ 'habitation ${ }^{13}$ dont les retraités peuvent bénéficier.

La contribution positive de la taxe foncière aux inégalités représente probablement un effet correcteur des différences entre les propriétaires et les locataires. La taxe foncière révèle une inégalité de niveau de vie: celle ayant trait aux dépenses de logement. Sa contribution positive peut être assimilée à la différence de pouvoir d'achat entre les propriétaires et les locataires.

Tableau 3 Résultats du GMR, 2007

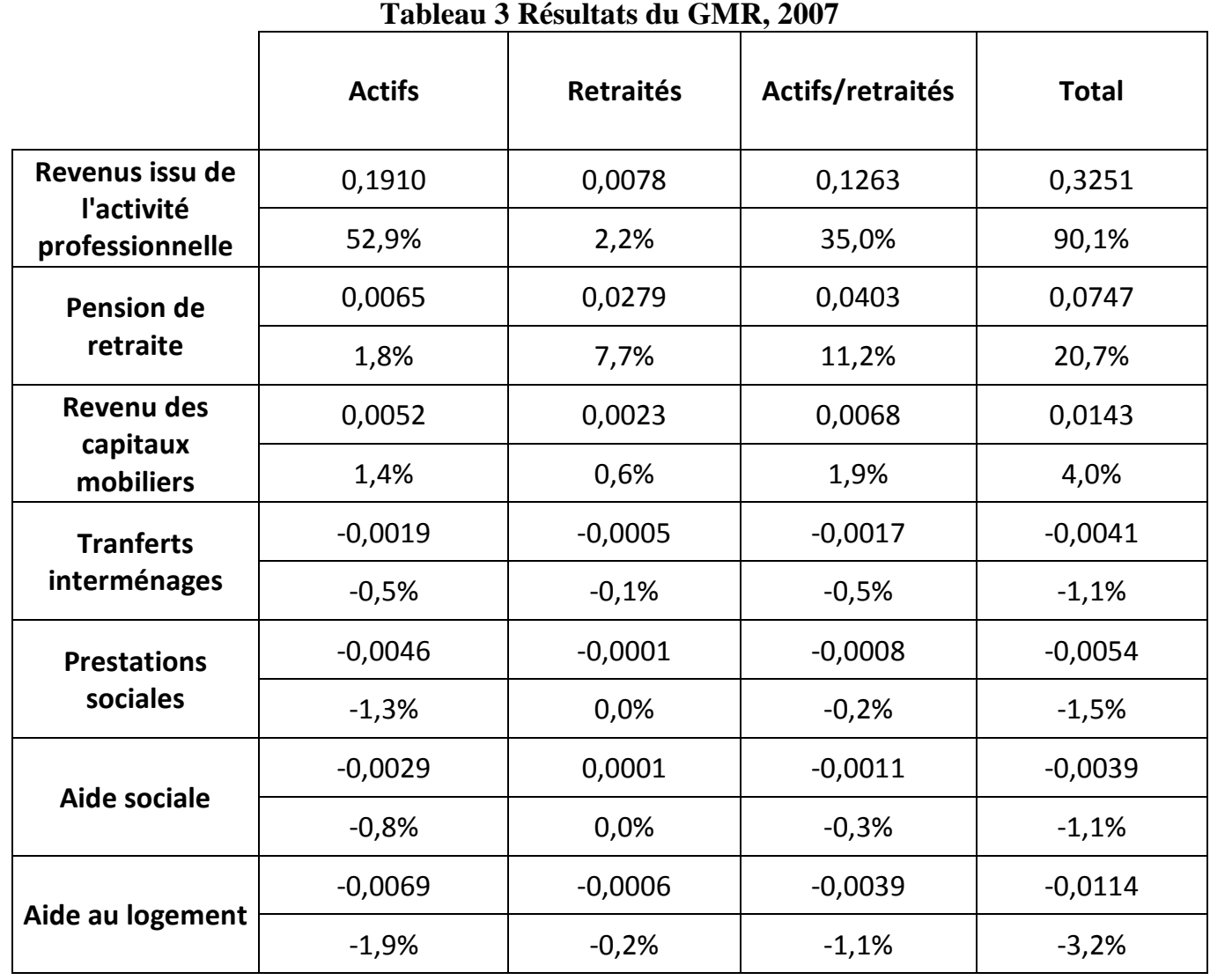

\footnotetext{
${ }^{12}$ Les pensionnés peuvent également bénéficier de majorations du nombre de parts de quotient familial lorsqu'ils sont titulaires de la carte de combattant, d'une pension militaire d'invalidité ou sont des victimes de guerre, et ce sous condition d'âge.

${ }^{13}$ La non imposition concerne les pensionnaires de maisons de retraite n'ayant pas le libre usage de leur logement. Les personnes âgées de plus de 60 ans peuvent être exonérées, sous condition de ressource, de la taxe d'habitation. Pour bénéficier de cet avantage fiscal, elles doivent néanmoins ne vivre qu'avec les personnes prévues par le code de Sécurité Sociale. Si ces contribuables de plus de 60 ans occupent leur logement avec un ou plusieurs enfants majeurs demandeurs d'emploi, ils bénéficient d'un dégrèvement d'office.
} 


\begin{tabular}{|c|c|c|c|c|}
\hline \multirow{2}{*}{$\begin{array}{l}\text { Prestations } \\
\text { maladie }\end{array}$} & 0,0011 & 0,0003 & 0,0013 & 0,0026 \\
\hline & $0,3 \%$ & $0,1 \%$ & $0,3 \%$ & $0,7 \%$ \\
\hline \multirow{2}{*}{$\begin{array}{l}\text { Allocations } \\
\text { chômage }\end{array}$} & $-0,0004$ & 0,0007 & 0,0022 & 0,0025 \\
\hline & $-0,1 \%$ & $0,2 \%$ & $0,6 \%$ & $0,7 \%$ \\
\hline \multirow{2}{*}{$\begin{array}{l}\text { Pension de } \\
\text { réversion }\end{array}$} & 0,0000 & $-0,0001$ & $-0,0003$ & $-0,0004$ \\
\hline & $0,0 \%$ & $0,0 \%$ & $-0,1 \%$ & $-0,1 \%$ \\
\hline \multirow{2}{*}{$\begin{array}{l}\text { Prestations } \\
\text { invalidité }\end{array}$} & $-0,0001$ & 0,0006 & 0,0012 & 0,0018 \\
\hline & $0,0 \%$ & $0,2 \%$ & $0,3 \%$ & $0,5 \%$ \\
\hline \multirow{2}{*}{$\begin{array}{l}\text { Pension issue } \\
\text { d'un régime de } \\
\text { retraite privé }\end{array}$} & 0,0001 & 0,0000 & 0,0001 & 0,0002 \\
\hline & $0,0 \%$ & $0,0 \%$ & $0,0 \%$ & $0,1 \%$ \\
\hline \multirow{2}{*}{$\begin{array}{l}\text { Impôt sur la } \\
\text { fortune }\end{array}$} & $-0,0001$ & $-0,0001$ & $-0,0003$ & $-0,0005$ \\
\hline & $0,0 \%$ & $0,0 \%$ & $-0,1 \%$ & $-0,1 \%$ \\
\hline \multirow{2}{*}{$\begin{array}{l}\text { Impôt sur le } \\
\text { revenu et taxe } \\
\text { d'habitation }\end{array}$} & $-0,0179$ & $-0,0041$ & $-0,0170$ & $-0,0390$ \\
\hline & $-5,0 \%$ & $-1,1 \%$ & $-4,7 \%$ & $-10,8 \%$ \\
\hline \multirow{2}{*}{ Taxe foncière } & 0,0008 & 0,0009 & 0,0026 & 0,0043 \\
\hline & $0,2 \%$ & $0,2 \%$ & $0,7 \%$ & $1,2 \%$ \\
\hline \multirow{2}{*}{$\begin{array}{l}\text { Total/revenu } \\
\text { disponible }\end{array}$} & 0,17 & 0,04 & 0,16 & 0,36 \\
\hline & $47,1 \%$ & $9,7 \%$ & $43,1 \%$ & $100,0 \%$ \\
\hline
\end{tabular}

Source : SRCV, calcul de l'auteur

\section{Conclusion}

Le GMR nous permet de lire les effets de la politique redistributive en France, mais ne nous permet pas de tirer des conclusions quant à l'efficacité de la politique menée par le décideur public. N'ayant pas confronté les résultats obtenus avec les objectifs affichés, nous ne pouvons tirer des conclusions en termes d'évaluation des politiques économiques. Néanmoins, en établissant une cartographie des inégalités, il permet de mieux cibler les populations souhaitées lors de la mise en place des politiques de lutte contre les inégalités. Le GMR peut s'avérer utile en amont du choix de politique économique.

Les prestations et prélèvements fiscaux entraînent une baisse marquée des inégalités parmi les actifs. Si les actifs ne percevaient pas de prestations sociales, l'indicateur d'inégalités serait 1,3\% plus élevé. L'impôt sur le revenu et la taxe d'habitation acquittés par les actifs permettent également de diminuer l'indicateur d'inégalité de 5,0\% en 2007. Ces politiques se justifient par une contribution particulièrement importante des inégalités entre actifs aux inégalités totales. 
Le versement des pensions explique près de $21,0 \%$ des inégalités en 2007 , mais les retraités sont sous représentés dans l'indicateur d'inégalité globale relativement à leur proportion dans la population.

Le GMR nous fournit une cartographie des inégalités dans le cadre d'une réflexion sur les inégalités actifs/retraités. Son caractère positif et descriptif permet d'obtenir des informations précises et détaillées. Néanmoins, certains aspects de l'outil semblent difficiles à interpréter comme par exemple la contribution croisée des actifs et des retraités à l'inégalité totale.

Les travaux développés dans le présent article pourront notamment être complétés à des fins utiles par une analyse de l'impact de la fiscalité préférentielle à destination des retraités en matière d'inégalités, dans un contexte de réforme où les retraités ont désormais un niveau de vie équivalent aux ménages actifs avec enfants (El Mekkaoui De Freitas et al., 2008 ; COR, 2009). 


\section{Bibliographie}

ANDRIEUX V. \& CHANTEL C. [2011], " Les retraites perçues fin 2008 », Etudes et résultats 758, Drees

ATKINSON A.B. [1970], "On measurement of economic inequality", Journal of Economic Theory, 2, 3, 244-263,

ATKINSON T., GLAUDE M., OLIER L. \& PIKETTY T. [2001], Inégalités économiques, Rapport du Conseil d'Analyse Economique,

BARR N. [2001], The Welfare State as Piggy Bank, Information, Risk, Uncertainty, and the role of the State, Oxford: Oxford University Press, 320p,

BOURGUIGNON F. [1998], Fiscalité et Redistribution, Rapport du Conseil d'Analyse Economique,

BROWN S.G. \& PRUS R.L, [2006], "Income inequality over the later-life course: a comparative analysis of seven OECD countries", SEDAP Research paper $n^{\circ} 154$,

BURRICAND C. \& DELOFFRE A. [2007], "L'évolution des retraites verses entre 2000 et 2004 », Etudes et résultats 556, Drees,

COWELL, F.A. [1995] Measuring Inequality (2nd edition). Harvester Wheatsheaf, Hemel Hempstead.

Conseil d'orientation des retraites [2009] Niveaux de vie comparés des retraités et des actifs : évolutions récentes, Document de travail pour la séance plénière du 21 octobre 2009,

DONALDSON D., \& WEYMARK J.A [1980] "Ethically flexible Gini indices for income distribution in the continuum", Journal of Economic Theory, 29, 353-358,

DUCAMIN B., BACONNIER R. \& BRIET R. [1996], Étude des prélèvements fiscaux et sociaux pesant sur les ménages: rapport au ministre du budget, La Documentation Française, collection des rapports officiels,

EBERT, U [2010] "The decomposition of inequality reconsidered: Weakly decomposable measures", Mathematical Social Sciences, 60, 94-103,

EL MEKKAOUI DE FREITAS N., BRIARD K., DUC C., LEGENDRE B., MAGE S. [2008], Aléas de Carrière, inégalités et retraite, Rapport de Recherche $n^{\circ} 47$ CEE, 229 pages, Juin.

GINI C. [1921], "Measurement of inequality of incomes", The Economic Journal, 31, 121, 124126 , 
LERMAN R.I. \& YITZHAKI S. [1985], "Income inequality effects by income source: A new approach and applications to the United States", The review of Economics and Statistics, 67, 151-156,

LITCHFIELD J., [1999] « Inequality: Methods and Tools », World Bank :

http://siteresources.worldbank.org/INTPGI/Resources/Inequality/litchfie.pdf

MUSSARD S. [2004], Décompositions multidimensionnelles du rapport moyen de Gini. Application aux revenus italiens de 1989 et 2000, Thèse de Doctorat, Université de Montpellier 1,

MUSSARD S. \& TERRAZA M. [2009], « Décompositions des mesures d'inégalités : le cas des coefficients de Gini et d'entropie ", Recherches économiques de Louvain, 75, 151-181.

Conseil des Prélèvements Obligatoires (2008), La répartition des prélèvements obligatoires entre générations et la question de l'équité intergénérationnelle, Rapport.

SAUTORY, O. [1996], "Les principales mesures d'inégalités ", Insee Méthodes 69-70-71, 235-267, Contribution aux Journées de Méthodologie Statistique.

SHORROCKS, A. F. [1980], "The Class of Additively Decomposable Inequality Measures", Econometrica, 48, 613-625.

THEIL H. [1967], Economics and Information Theory, North-Holland Publishing Company, Amsterdam, 488p. 


\section{Annexes}

\section{$\underline{\text { Annexe A : Détails du calcul du GMR }}$}

$$
G M R_{j j}=\sum_{i=1}^{n_{j}} \sum_{r=1}^{n_{j}} \frac{\left|x_{i j}-x_{r j}\right|}{n_{j}^{2} \max \left(x_{i j}, x_{r j}\right)}
$$

(Eq. 2 dans l'article)

On peut écrire : $\left|x_{i j}-x_{r j}\right|=\sum_{l=1}^{q}\left(x_{i j}^{l}+x_{r j}^{l}-2 x_{j, i r}^{* l}\right)$

En effet, si $\left.x_{i j}\right\rangle x_{r j}$, alors :

$\left|x_{i j}-x_{r j}\right|=x_{i j}-x_{r j}=\sum_{l=1}^{q}\left(x_{i j}^{l}-x_{r j}^{l}\right)=\sum_{l=1}^{q}\left(x_{i j}^{l}+x_{r j}^{l}-2 \min \left(x_{i j}^{l}, x_{r j}^{l}\right)\right)=\sum_{l=1}^{q}\left(x_{i j}^{l}+x_{r j}^{l}-2 x_{r j}^{l}\right)$

Puis si $x_{i j}<x_{r j}$, alors on a :

$\left|x_{i j}-x_{r j}\right|=x_{r j}-x_{i j}=\sum_{l=1}^{q}\left(x_{r j}^{l}-x_{i j}^{l}\right)=\sum_{l=1}^{q}\left(x_{i j}^{l}+x_{r j}^{l}-2 \min \left(x_{i j}^{l}, x_{r j}^{l}\right)\right)=\sum_{l=1}^{q}\left(x_{i j}^{l}+x_{r j}^{l}-2 x_{i j}^{l}\right)$

$$
G M R_{j h}=\sum_{i=1}^{n_{j}} \sum_{r=1}^{n_{h}} \frac{\left|x_{i j}-x_{r h}\right|}{n_{j} n_{h} \max \left(x_{i j}, x_{r h}\right)} \text { (Equation } 3 \text { dans I'article) }
$$

On peut écrire : $\left|x_{i j}-x_{r h}\right|=\sum_{l=1}^{q}\left(x_{i j}^{l}+x_{r h}^{l}-2 x_{j h, i r}^{*_{l}^{l}}\right)$

En effet, si $\left.x_{i j}\right\rangle x_{r h}$, alors :

$\left|x_{i j}-x_{r h}\right|=x_{i j}-x_{r h}=\sum_{l=1}^{q}\left(x_{i j}^{l}-x_{r h}^{l}\right)=\sum_{l=1}^{q}\left(x_{i j}^{l}+x_{r h}^{l}-2 \min \left(x_{i j}^{l}, x_{r h}^{l}\right)\right)=\sum_{l=1}^{q}\left(x_{i j}^{l}+x_{r h}^{l}-2 x_{r h}^{l}\right)$

Puis si $x_{i j}<x_{r h}$, alors on a :

$\left|x_{i j}-x_{r h}\right|=x_{r h}-x_{i j}=\sum_{l=1}^{q}\left(x_{r h}^{l}-x_{i j}^{l}\right)=\sum_{l=1}^{q}\left(x_{i j}^{l}+x_{r h}^{l}-2 \min \left(x_{i j}^{l}, x_{r h}^{l}\right)\right)=\sum_{l=1}^{q}\left(x_{i j}^{l}+x_{r h}^{l}-2 x_{i j}^{l}\right)$

De là, il vient : 


$$
\begin{aligned}
& G M R(x)=\sum_{l=1}^{q} \sum_{j=1}^{k} \sum_{i=1}^{n_{j}} \sum_{r=1}^{n_{j}} z_{j} \frac{x_{i j}^{l}+x_{r j}^{l}-2 \min \left(x_{i j}^{l}, x_{r j}^{l}\right)}{n_{j}^{2} \max \left(x_{i j}, x_{r j}\right)}+2 \sum_{l=1}^{q} \sum_{j=2}^{k} \sum_{h=1}^{j-1} \sum_{i=1}^{n_{j}} \sum_{r=1}^{n_{j}} z_{j h} \frac{x_{i j}^{l}+x_{r h}^{l}-2 \min \left(x_{i j}^{l}, x_{r h}^{l}\right)}{n_{j} n_{h} \max \left(x_{i j}, x_{r h}\right)} \\
& \Leftrightarrow \operatorname{GMR}(x)=\sum_{l=1}^{q} \sum_{j=1}^{k} \sum_{i=1}^{n_{j}} \sum_{r=1}^{n_{j}} z_{j} \frac{x_{i j}^{l}+x_{r j}^{l}-2 x_{j, i r}^{* l}}{n_{j}^{2} \max \left(x_{i j}, x_{r j}\right)}+2 \sum_{l=1}^{q} \sum_{j=2}^{k} \sum_{h=1}^{j-1} \sum_{i=1}^{n_{j}} \sum_{r=1}^{n_{j}} z_{j h} \frac{x_{i j}^{l}+x_{r h}^{l}-2 x_{j h, i r}^{* l}}{n_{j} n_{h} \max \left(x_{i j,}, x_{r h}\right)} \\
& \text { Où : } x_{j, i r}^{* l}=\min \left(x_{i j}^{l}, x_{r j}^{l}\right) \text { et } x_{j h, i r}^{* l}=\min \left(x_{i j}^{l}, x_{r h}^{l}\right)
\end{aligned}
$$

\section{Annexe B : Calcul du revenu disponible}

Les sources de revenu propres aux individus sont additionnées au sein de chaque ménage (Cf. Schéma 1 ci-après) :

- les rémunérations salariales, revenus d'activité des indépendants, et l'autoconsommation, regroupés en une seule variable pour notre décomposition,

- les indemnités maladies,

- les allocations chômage,

- les pensions d'invalidité,

- les pensions de retraite (pension de base et pension complémentaire),

- et les retraites issues des régimes privés, que nous ajoutons bien que le calcul du revenu disponible dans l'enquête ne les inclut pas. L'importance de cette source de revenu est toutefois négligeable car ne concerne que peu d'individus de notre échantillon.

A ces sources individuelles de revenu sont additionnées les variables propres aux ménages (Cf. Schéma 1) :

- les prestations familiales, auxquelles nous ajoutons les transferts nets entre ménages (pensions alimentaires, loyers perçus et reçus, etc.), les bourses scolaires et bourses d'études individuelles,

- les aides sociales (revenu minimum d'insertion, etc.),

- les aides au logement,

- les revenus des capitaux mobiliers,

- l'impôt de solidarité sur la fortune, soustrait des autres revenus,

- l'impôt sur le revenu et la taxe d'habitation, également soustraits,

- enfin la taxe foncière, que nous choisissons d'ôter des autres sources de revenus, bien qu'elle soit incluse dans la définition du revenu disponible. 
Tableau 4 Taille des échantillons considérés, 2007

\begin{tabular}{|c|c|}
\cline { 2 - 2 } & Effectif \\
Actifs & 11455 \\
Retraités & 5081 \\
$\begin{array}{c}\text { Rapport } \\
\text { démographique } \\
\text { entre retraités et } \\
\text { actifs }\end{array}$ & $44 \%$ \\
\hline \multicolumn{2}{|c|}{ Source : SRCV }
\end{tabular}

Schéma 1 : les étapes de distribution des revenus

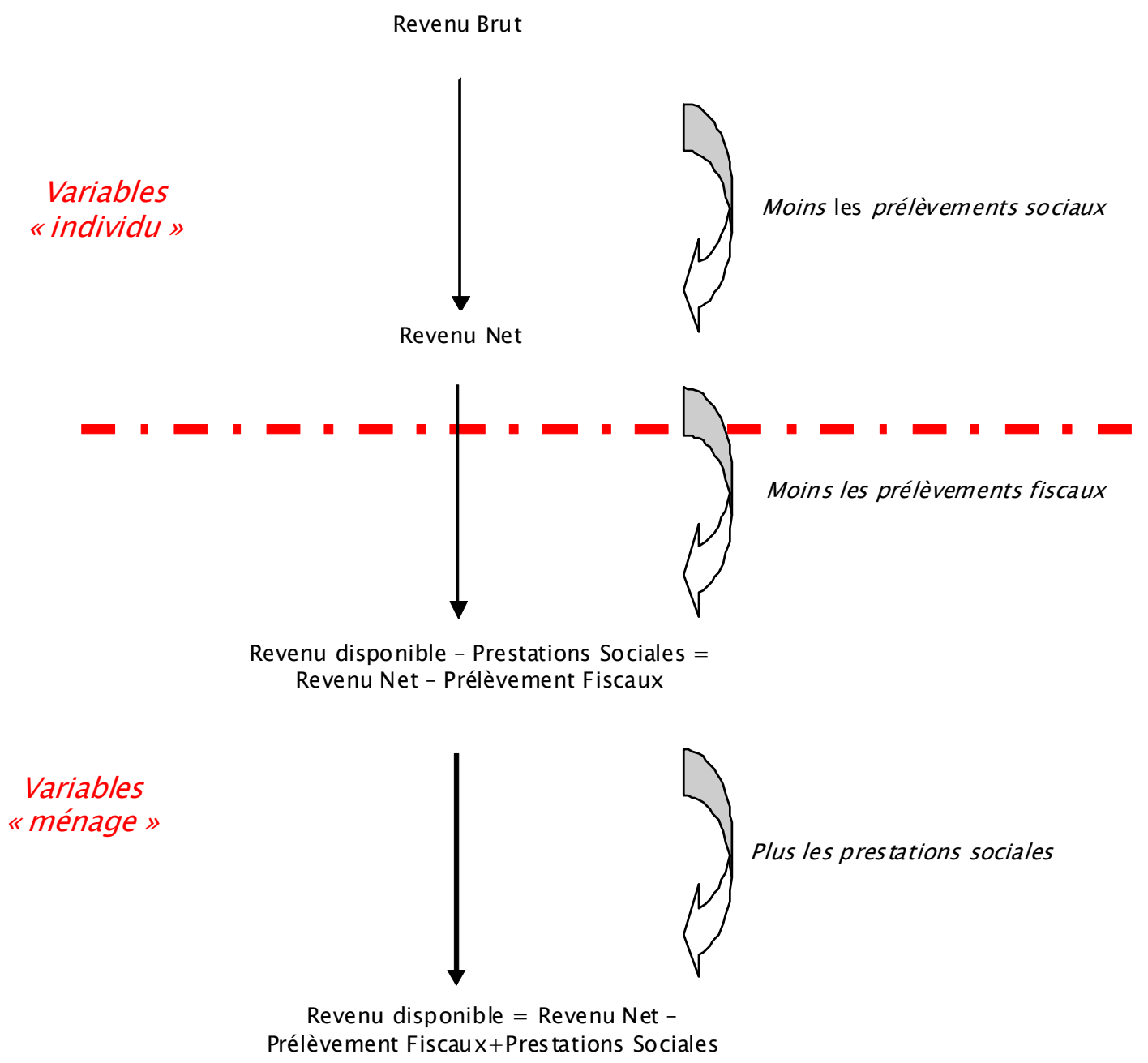

Source : Auteur 